\title{
Inter-cell interference mitigation using adaptive reduced power subframes in heterogeneous networks
}

\author{
Mohammed I. Aal-nouman ${ }^{1}$, Osamah Abdullah ${ }^{2}$, Noor Qusay A. AlShaikhli ${ }^{3}$ \\ ${ }^{1,3}$ College of Information Engineering, Al-Nahrain University, Baghdad, Iraq \\ ${ }^{2}$ Department of Electrical Power Engineering Techniques, Al-Ma'moon University College, Baghdad, Iraq
}

\begin{tabular}{l} 
Article Info \\
\hline Article history: \\
Received Sep 9, 2020 \\
Revised Dec 1, 2020 \\
Accepted Jan 13, 2021 \\
\hline
\end{tabular}

Keywords:

Almost blank subframes Heterogeneous networks Inter-cell interference SINR

\begin{abstract}
With the remarkable impact and fast growth of the mobile networks, the mobile base stations have been increased too, especially in the high population areas. These base stations will be overloaded by users, for that reason the small cells (like pico cells) were introduced. However, the intercell interference will be high in this type of Heterogeneous networks. There are many solutions to mitigate this interference like the inter-cell interference coordination (ICIC), and then the further enhanced ICIC (Fe-ICIC) where the almost blank subframes are used to give priority to the (victim users). But it could be a waste of bandwidth due to the unused subframes. For that reason, in this paper, we proposed an adaptive reduced power subframe that reduces its power ratio according to the user's signal-to-interference-plus-noise ratio (SINR) in order to get a better throughput and to mitigate the intercell interference. When the user is far from the cell, the case will be considered as an edge user and will get a higher priority to be served first. The results show that the throughput of all users in the macro cells and pico cell will be improved when applying the proposed scheme in term of throughput for the users and the cells.
\end{abstract}

This is an open access article under the CC BY-SA license.

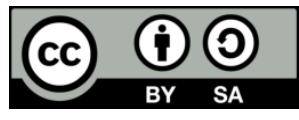

\section{Corresponding Author:}

Mohammed Imad Aal-Nouman

Department of Information and communications Engineering

College of Information Engineering, Al-Nahrain University

Al-Jadryah, Baghdad, Iraq

Email: m.aalnouman@coie-nahrain.edu.iq

\section{INTRODUCTION}

The Heterogeneous network in the long-term evolution (LTE) and LTE-Advanced (LTE-A) is considered as one of the solutions that were introduced by the $3^{\text {rd }}$ generation partnership project (3GPP) to increase the network coverage and increase its capacity. However, because of the co-channel and multi cells in such networks, the Inter-cell interference (ICI) can be considered as an issue. Generally, the user and the cell throughput of the whole network will be affected negatively and this negative impact will affect the edge user mostly. The main components of the Heterogeneous network can be listed as: Macro cell, pico cell, femto cell, and the user equipment (UE) which can also be classified according to the serving cell, pico cell user (PUE), macro cell user (MUE) and femto cell user (FUE). Moreover, the users can also be classified according to their location within the cell, so if the user is in the center of the cell coverage, the case will be considered as a normal user, but if the user is at the edge of the cell coverage then the case will be considered as an edge user [1]. Figure 1 shows the main components of the Heterogeneous network.

As shown in Figure 1, the main macro cell will add an interference to the low-power cell users (LPCs), the green line is showing the serving cells connection while the red line showing the interferences 
from the macro cell. Usually, the UE will select the cell with a maximum reference signal received power (RSRP) among the surrounding cells. However, in some cases and because of the differences in the power levels in the heterogonous network, the UE may select the macro cell as a serving cell because it has the highest power, and this leaves the small cells unused. This may cause interference to the other small cells in the network [2]. To solve this problem, the cell range expansion (CRE) was introduced in order to get a balance to the network. The concept of the CRE is adding the cell-specific offset (CSO) to the reference signal received power (RSRP) of the small cells to get larger coverage area and to serve more users. But the cell expanded areas will still have an interference due to macro cell high power. Figure 2 shows the CRE region (the gray area) [3].

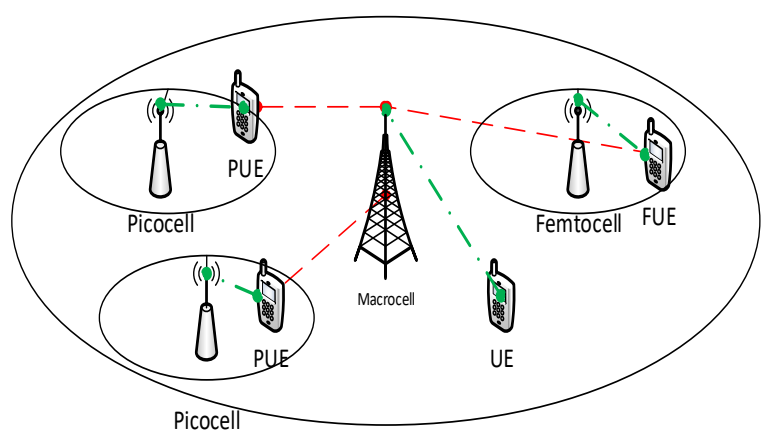

Figure 1. Components of heterogeneous networks

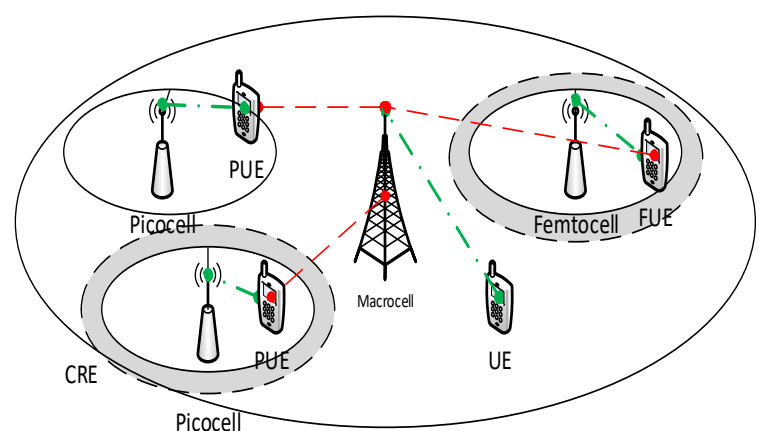

Figure 2. Cell range expansion

From release 10, the third-generation partnership project (3GPP) introduced the time-domain intercell interference coordination (TD-ICIC) schemes. In this proposal, the interference caused by the multichannels will be mitigated and the power of the macro cell will be lowered in certain subframes to let the other small cells use these subframes [2]. When using the TD-ICIC method, the transmission power of some subframes of the macro cell within the resource blocks will be reduced to minimum in the CRE, to reduce the interference to the victim UEs, this type of subframe is called almost blank subframes (ABS) [4-8]. Figure 3 shows these types of subframes.

The scheme works by muting the macro cell in certain subframes namely (ABS), and give the priority to other cells to use a protected subframe to avoid the interferences that may be caused by the macro cells to the other cells. Where the $\mathrm{N}$ is the normal subframe, ABS is the almost blank subframe in which the macro cell gets muted or uses a very low power, and the PSF is the protected subframe. These ABS subframes are usually used only for common signals (control, page signal, broadcast signals) by the macro cells $[9,10]$ and using these subframes along with CRE will improve the throughput of the cell edge users as long as the macro cell is silent or reduced power [7, 11].

The main problem that this paper tries to address is to mitigate the inter cell interference in the heterogeneous networks by utilizing the subframes of the macro and pico cells. So, we proposed a novel scheme to solve the problem, our contribution is the design and implementation of an adaptive ICIC that adaptively change the percentage of power in the (new version) of the almost blank subframes based on the priority of the UE in the queue according to its signal to interference plus noise ratio and its distance from the serving cell.

\begin{tabular}{|c|c|c|c|c|c|c|c|c|c|}
\hline \multicolumn{10}{|c|}{ Macrocell } \\
\hline $\mathrm{N}$ & ABS & $\mathrm{N}$ & ABS & $\mathrm{N}$ & $A B S$ & $\mathrm{~N}$ & ABS & $\mathrm{N}$ & ABS \\
\hline$N$ & PSF & $\mathrm{N}$ & PSF & $\mathrm{N}$ & PSF & $N$ & PSF & $\mathrm{N}$ & PSF \\
\hline
\end{tabular}

Figure 3. Subframe example showing the (ABS) in Heterogeneous networks

The proposed work in this paper is special and different than the other authors' works in several points:

- We proposed special subframes that can adaptively change their percentage of the power while other systems usually have a fixed power. 
- We give the priority to the cell-edge users while other schemes give the user's priority based on the scheduler they used.

- We take the SINR as the main element to be checked to give the priority to the users, other works also used it but many use the SNR (signal to noise ratio) only.

The rest of the paper is organized as follows: Section 2 will show the related works, section 3 will show the proposed method, section 4 will show the results and it's followed by the discussion in section 5 . Finally, the conclusions will be presented in section 6 .

\section{RELATED WORK IN ICIC}

The idea of using CRE has attracted the researchers to improve the Heterogeneous network performance. Many researchers change the CRE region depending on signal-to-interference-plus-noise ratio (SINR) like in $[7,12]$ and other researchers suggested to extend the region based on the analysis and update of the CSO as proposed [13]. Then the TD-ICIC was introduced by 3GPP to reduce the interferences from multi-cells toward pico cells and femto cells in the Heterogeneous networks by using the ABS [14], in which the macro cell is abstained from transmission in this period of subframes because these subframes are protected to be used by cell-edge users who are using the same frequency band [15, 16]. To control and coordinate these subframes, a scheduler has to be used like the proposal that is introduced by [17]. The researchers used a decentralized resource management framework by using the dynamic fractional frequency reuse (FFR) to coordinate the inter-cell interference between the UEs and give a priority to the edge users. The throughput of the low power cells generally and the cell-edge specifically will be enhanced when using the ABS scheme as can be seen in $[9,15,16]$

TD-ICIC scheme has been chosen by [7] to be used along with extended version of CRE, which divided the CRE region into inner and outer areas. The whole bandwidth has been shared with the macro cells and the low power cells (Pico cells), the author used the reduced power subframe (RPS) along with the almost blank subframe (ABS). This will keep an optimal performance for the Pico cells while keeping the Heterogeneous network performance balanced.

In release $11 \mathrm{LTE}$, the $3 \mathrm{GPP}$ proposed the new scheme which is the further enhanced ICIC (FeICIC) $[18,19]$. The scheme will reduce the power of some subframes for a specific node instead of blank it totally, such subframes are called low power ABS (LP-ABS) which will give a better efficiency to the network. The scheme has been used by [19], it gives a higher priority to the UE in the center of the macro cells. These schemes allow to decrease the power to $30 \mathrm{dBm}$ as mentioned in $[20,21]$ to give a better performance to the edge users in the macro cells. The value of power level is not constant and can be variable which depends on many factors, like ABS power ratio, the value of extended region in CRE and the model of the channel. However, the value of $30 \mathrm{dBm}$ has always been used by the researcher even though it is not always the optimum value [22-24]. Because of the fact that the network performance can be changed according to the power level in the LP-ABS and the extended area of the CRE, authors of [25] proposed an adaptive ICIC based on FFR. Also, the authors of [26] introduced a method where the small cells can organize themselves in the network to reduce the interferences by suggesting a dynamic FFR (DFFR) method.

The authors of [2] also suggest a mechanism that changes the power of ABS dynamically to get a better quality of service (QoS). The authors used the traffic mode that supports the multimedia communications only. Different cell-selection offsets (CSOs) has been suggested by [27] to change the coverage of the CRE area. The CSO is changed adaptively in order to improve the performance of the Heterogeneous network. The authors in [28] suggested a new novel idea that changes the CRE area and the ABS dynamically based on the cell traffics of each cell in the Heterogeneous network [28]. Some researchers tried to get a better performance for the user in the $5 \mathrm{G}$ system like in [29] where they investigated the interference coordination in massive MIMO and how that will be affected by the transfer from $4 \mathrm{G}$ to $5 \mathrm{G}$ network. Also the authors in [30] suggested many techniques to mitigate the inter cell interference: ICIC of pilot sequence configuration for cell-edge users, coordinated small cell DTX for the downlink and on-demand power boosting and coordinated muting. From the above literature, it is obvious that CRE and the change of the power rate in the ABS and LP-ABS can increase the throughput of the cells, the users in the cells and the edge users as well. From that we suggest an adaptive power ABS that can be changed according to the distance between the user and the cell which will change the value of the SINR, and give a higher priority to the edge user in the scheduler.

\section{PROPOSED WORK}

The user throughput calculation is based mainly on many factors, and one of these factors is the SINR, which is used to calculate the signal to interference ratio and take the noise in account [31, 32]. Also, the distance plays a role in the SINR value that when the distance between the UE and serving cell is

Inter-cell interference mitigation using adaptive reduced power subframes... (Mohammed I. Aal-nouman) 
increased, the interferences from other cells will be increase too. The SINR of UE (y) that is connected to a cell (x) can be calculated as in (1) [7, 33-35].

$$
\gamma_{x, y}=\left(\frac{P_{x}^{M} \cdot H_{x, y}}{\sum_{q \neq x}^{I} P_{q} \cdot H_{q \cdot y}+N_{0}}\right)
$$

where $P_{x}$ is the transmitting power of the attached cell (x), and $P_{q}$ is the transmitting power of the interfererneighbor cells. $H_{x, y}$ is the gain of the serving cells and $H_{q, y}$ is the accumulated gain of the serving plus the interferer cells from the $U E$ perspective view, and $N_{0}$ is the noise.

To find the SINR of a specific cell some modification should be applied so the macro cell SINR will be as shown in (2) [33-35].

$$
\gamma_{x, y}=\left(\frac{P_{x}^{M} \cdot H_{x, y}}{\sum_{n_{S=1}}^{N_{S}} P_{n_{S}} \cdot H_{n_{S} \cdot y}+\sum_{n_{Z=1}}^{N_{Z}} P_{n_{Z}} \cdot H_{n_{Z} \cdot y}+N_{0}}\right)
$$

where

$$
P_{x}^{M}= \begin{cases}P_{m} & \text {, if normal subframes are used } \\ P_{L P} & \text {, if RP subframes are used }\end{cases}
$$

Noting that, $P_{n_{S}}$ is the transmitting power from the neighbor macro cells and $P_{n_{z}}$ is the transmitting power of the neighbor Pico cells. While the, $H_{n_{s}, y}$ and $H_{n_{z}, y}$ denote the channel gains from both neighbor macro and pico cells respectively.

The SINR of a user (y) that is connected to a Pico cell (L) can have three different values according to the sub frame, shown in (4) [33-35]:

$$
\gamma_{L, y}=\left(\frac{P_{L}^{P} \cdot H_{L, y}}{\phi \cdot H_{x, y}+\sum_{n_{S=1}}^{N_{S}} P_{n_{m}} \cdot H_{n_{m} \cdot y}+\sum_{n_{Z=1}}^{N_{Z}} P_{n_{Z}} \cdot H_{n_{Z} \cdot y}+N_{0}}\right)
$$

Noting that $P_{L}^{P}$ is the transmitting power of the serving pico cell (L) and $H_{L, y}$ is the gain to the user (y), while the $\Phi$ is the transmitting power of the main macro cell.

$$
\Phi=\left\{\begin{array}{c}
P_{m}, \text { if the normal subframes are used } \\
P_{L P}, \quad \text { if the Reduced Power subframes are used } \\
0, \text { if almost Blank Subframes is used }
\end{array}\right.
$$

It is obvious that the value of $\Phi$ will change the value of the SINR of the Pico cell users and could be a higher SINR for that users who are near the edge of the cell and most of the interference signals are coming from the main macro cell, the minimum SINR threshold is $-2 \mathrm{~dB}$.

In this paper the urban model will be used. In this model, the wave propagation will have two major paths: over the rooftop when the user is far from the cell, and along the street which is considerable when the user is close to the serving cell [7,36]. The propagation model for the main cell can be expressed as in (5) [37].

$$
L=40 \cdot\left(1-4 * 10^{-3} \cdot H b\right) \cdot \log (R)-18 \cdot \log (H b)+21 \cdot \log (f)+80 d b
$$

where $f$ is the frequency in $\mathrm{MHz}, H b$ is the antenna height, $R$ is the distance in $\mathrm{Km}$. After applying the carrier frequency used in the paper $2 \mathrm{GHz}$, the antenna height is equal to 15 meter and the shadowing with standard of $8 \mathrm{~dB}$ will be added, the calculation of the pathloss of the macro cell will be as in $(6)[7,37]$.

$$
\text { pathloss }_{\text {macro }}=L+\log (f)
$$


Also, in a same way, the propagation model calculation of the pico cells for the urban areas will be as in $(7)[7,37]$.

$$
L=140.7+36.7 \log (R)
$$

So for that reason, we proposed an algorithm that uses TD-ICIC with adaptive rate of LP-ABS power. This power can be changed according to the distance from the serving cells in order to increase the throughput of the edge users in the pico cells. Figure 4 shows the proposed algorithm, where $\mathrm{N}$ is a normal subframe, ABS is the almost blank frame, and RP is the reduced power subframe where our proposed scheme will be changing the power adaptively.

Macrocell

\begin{tabular}{|l|l|l|l|l|l|l|l|l|l|}
\hline $\mathrm{N}$ & $\mathrm{ABS}$ & $\mathrm{RP}$ & $\mathrm{RP}$ & $\mathrm{RP}$ & $\mathrm{N}$ & $\mathrm{N}$ & $\mathrm{N}$ & $\mathrm{N}$ & $\mathrm{N}$ \\
\hline $\mathrm{N}$ & $\mathrm{N}$ & $\mathrm{N}$ & $\mathrm{N}$ & $\mathrm{N}$ & $\mathrm{N}$ & $\mathrm{N}$ & $\mathrm{N}$ & $\mathrm{N}$ & $\mathrm{N}$ \\
\hline
\end{tabular}

Figure 4. Proposed scheme subframes

In order to control and manage the resource block effectively, the schedullar has to give different priorities for the users depending on some factors. The (victim users) who are the useres that are located at the edge of the cell will have a higher priority, the schedullar will give the priority to the user with less SINR to use the subframe of the pico cells during the ABS and RP subframes of the macro cells, after that other users can connect and use the desired subframes. In this paper, we simulate the LTE-A HetGen network with a central eNodeB (evolved node B) that has three macro cells and two pico cells for each macro cell which gives a total of 6 pico cells. As can be seen in Figure 5, the X2 interface is used to connect these cells coordinating the interferences and to share the resources between them.

Different number of users are taken in different scenarios starting from 100, 200, 300, and 400, these UEs are distributed randomly in the network and set to attach the cell with the higher reference signal received power. We considered the interference that came from the neighboring macro cells. Then adding a bias of $3 \mathrm{~dB}$ for the $\mathrm{CRE}$ in each time to check the network behavior and the edge user throughput along with the cell throughput.

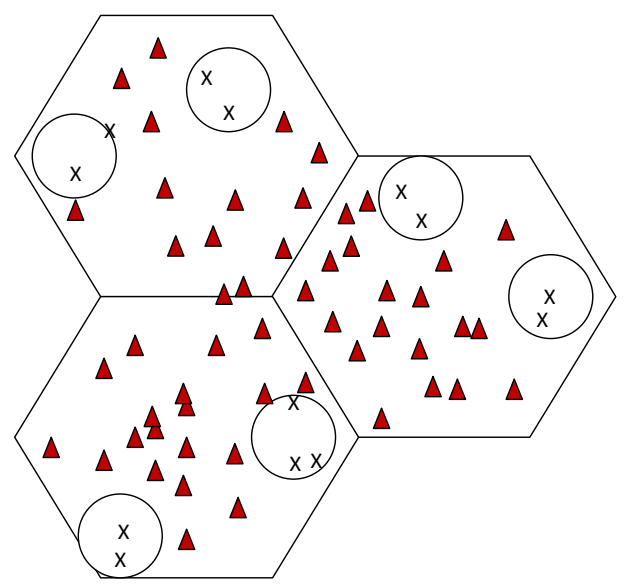

Figure 5. Heterogeneous network scenario

\section{RESULTS}

In this paper we used a system level simulation using a full-buffer for traffic mode. Different values of CRE have been taken in the results with different power ratios for the Reduced power subframes, the subframe takes a different ratio of power reduction starting from $10 \%$ to $90 \%$ with a step of $5 \%$, this ratio will depend on the SNIR of the user after sorting them in the queue before serving them. Figure 6 shows the

Inter-cell interference mitigation using adaptive reduced power subframes... (Mohammed I. Aal-nouman) 
average and edge user throughput for different power ratios (namely 25\%, 50\%, and 75\%) for the reduced power subframes with different CRE bias.

The figure shows that when reducing the power of the RP subframes in the macro cell the throughput for the edge users and average throughput for the whole UE in the pico cell can be improved. The reason of this is that there will be more power for the pico UE to use the subframes and also will be given a higher priority in the scheduler. For that we proposed a method to change the power of the users adaptively depending on the SINR, in which the power rate of the reduced power subframe can change accordingly, as can be seen in Figure 7.

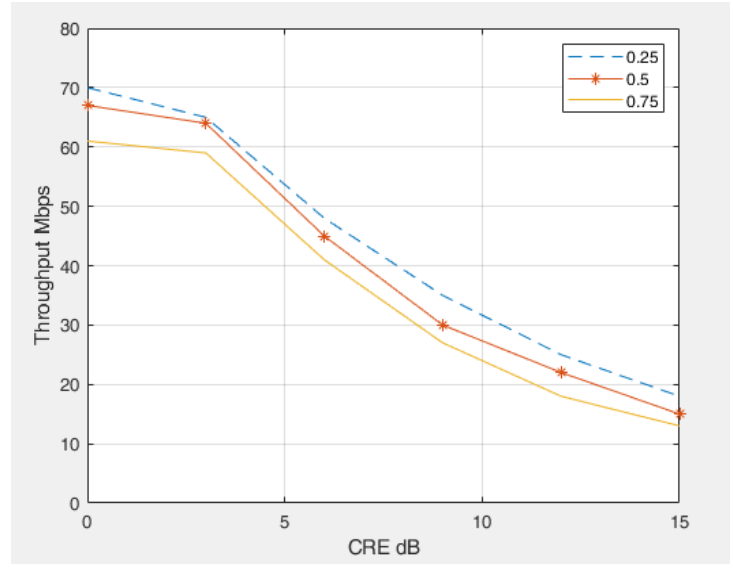

(a)

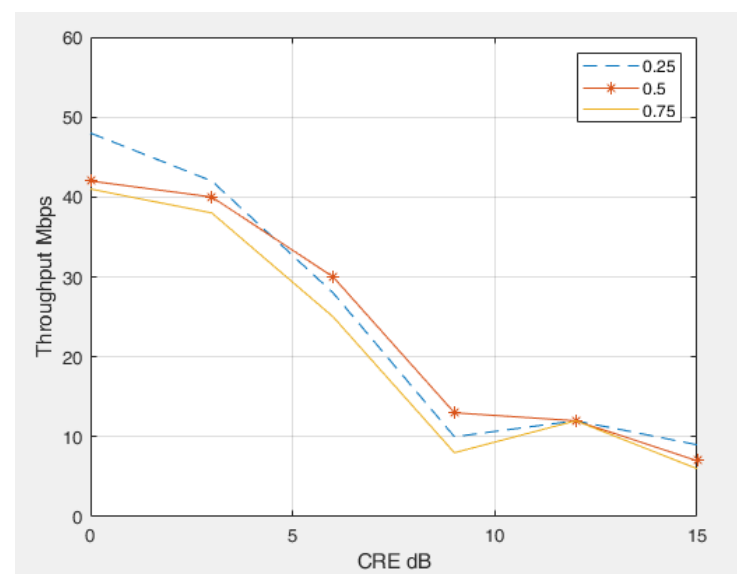

(b)

Figure 6. Pico cell users average throughput for; (a) all users, (b) edge user

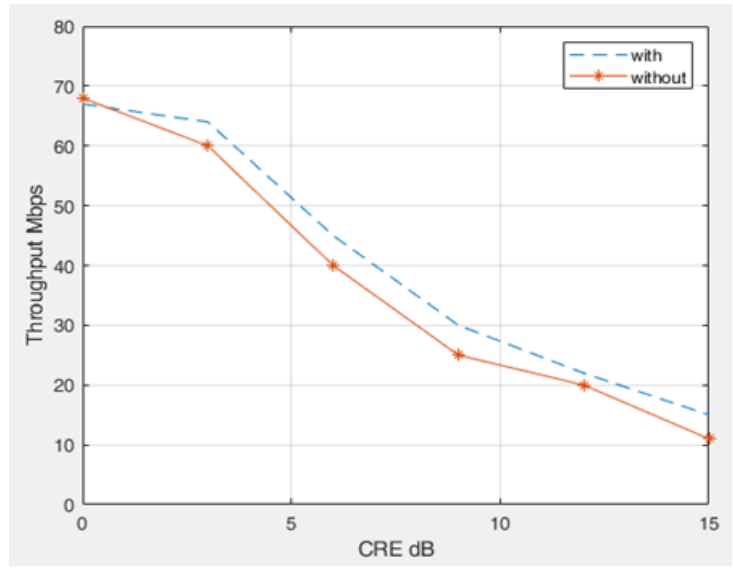

(a)

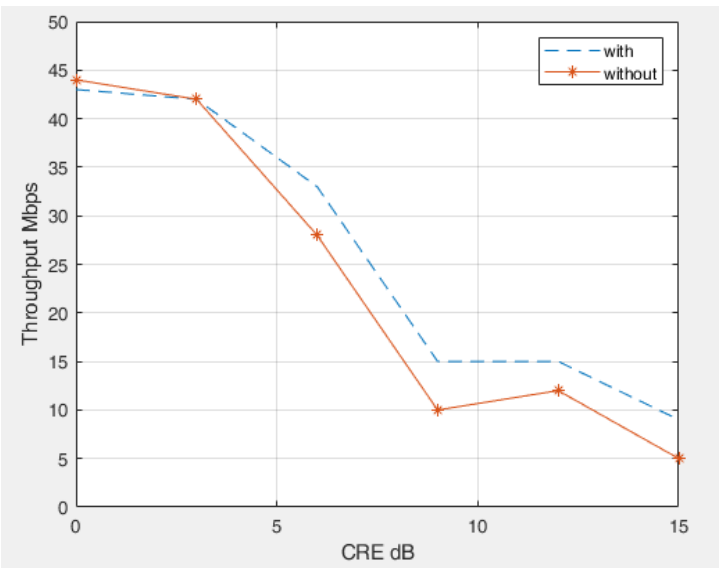

(b)

Figure 7. Throughput of the Pico cells users after and before applying the proposed scheme; (a) all UEs, (b) edge users

We can see the difference in the throughput for the UEs with and without the proposed scheme and it is clear that the throughput is improved when using the proposed scheme especially for the edge users. The macro cells are considered as the coordinator for many Pico cells and can share the resources between them. Figure 8 shows the throughput for the macro cell users with and without our scheme.

It can be clearly seen that the throughput of all users and the edge users in the macro cells have been improved. The edge users get better throughput because the scheduler has set a priority to them. Finally, the throughput of the total users in the networks can also be improved for the same reason as can be seen in Figure 9.

Finally, we take a different scenario to check the same user throughput with different distances from the cell, with and without using our proposed method and the results can be seen in Figure 10. As can be seen and because of the scheduler and due to the application of our proposed scheme, the user that has a higher 
distance from the serving cell will get a higher priority. For that reason, we can see a better performance for the users at the edge.

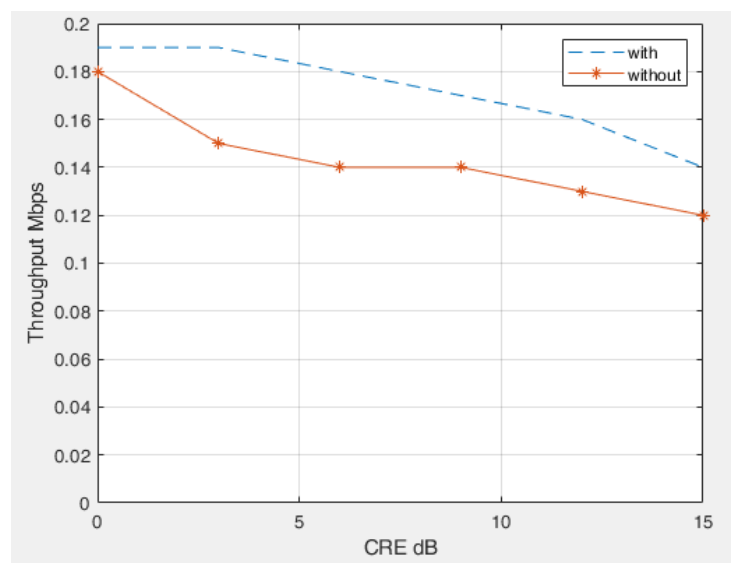

(a)

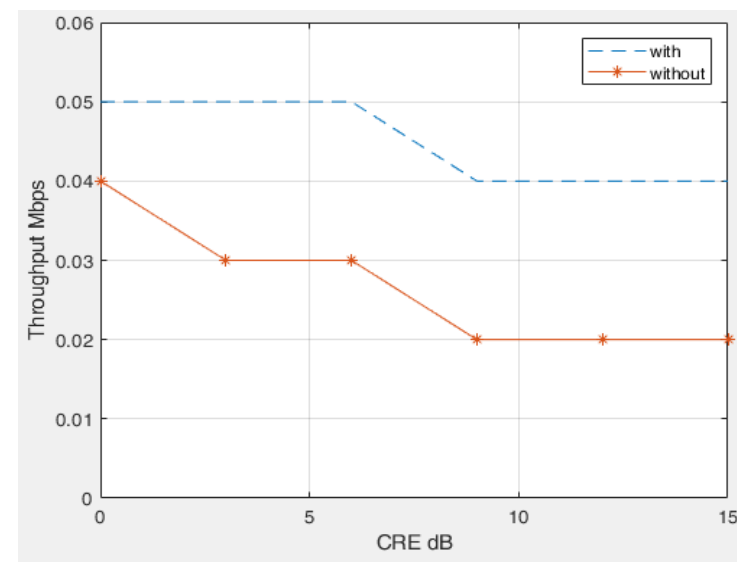

(b)

Figure 8. Macro UE Throughput before and after using the proposed scheme; (a) all users, (b) edge users

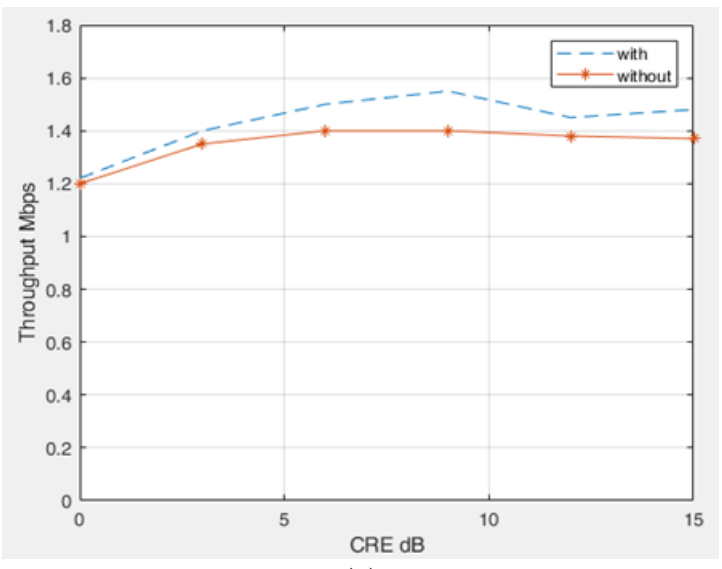

(a)

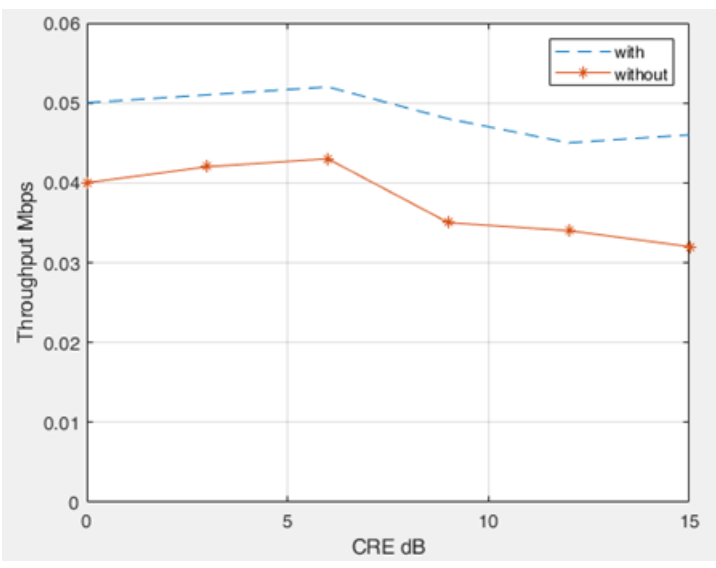

(b)

Figure 9. Total UE Throughput after and before the scheme; (a) all UE in the network,

(b) edge users in the network

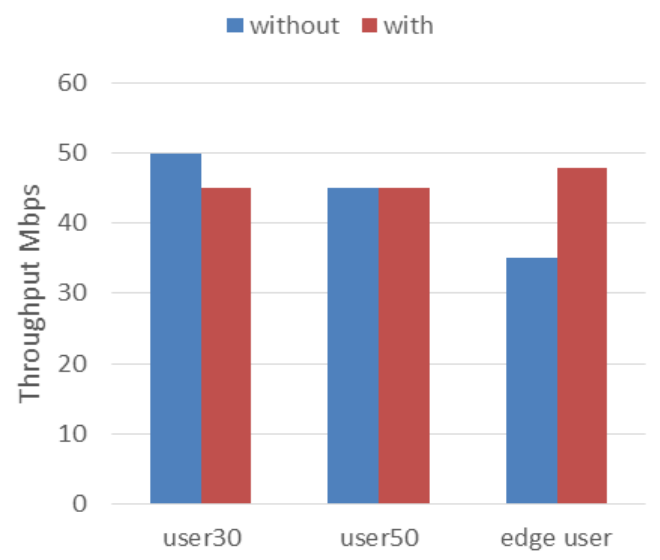

Figure 10. User throughput with different distances 


\section{DISCUSSION}

The paper proposed a shceme that reduces the power of special subfames in order to give more resources to the cell-edge users and give them a priority in the scheduler depending on their SINR. The reduced power subframes will change their power percentage depending on the value of the SINR, the ratios shown in the results are $(25 \%, 50 \%$, and $75 \%)$ while in the simulation the power ratios are taken from $(10 \%$ to $90 \%$ ) with a step of $5 \%$.

The results shown in this paper have four parts, firstly, we have to prove that the performance of the users will be affected if the power of the subframe has changed, so Figure 6 shows that the average throughput of the UEs in the pico cell will be improved when the power is lowered. On the other hand, the increased power in the subframe will improve the cell-edge user throughput for different values of the CRE. Secondly, we applied our proposed scheme, in which the power will be changed adaptively according to the SINR, so Figure 7 shows how the throughput has been improved for both the normal and the cell-edge users if we applied the scheme for pico cell, and the same situation if we applied the scheme for the macro cell as shown in Figure 8.

Thirdly, we took a bigger look for the whole network and as can be seen in Figure 9, all the UEs throughput has been improved. Finally, in Figure 10, we investigate what will happen if the user is far from the serving cell, to prove that the scheduler is giving a higher priority to the cell-edge users because they have a higher SINR, and then the users who are far from the cell but not considered as cell-edge users, and the lowest priority will be for those users who are near the serving cell because they will have a lower SINR in the network. The throughput improvement that is seen from the results is due to the adaptive change of the power percentage of the subframes, and thus will give the optimum scheduling to the users and consequently a better efficiency for the whole network.

\section{CONCLUSION}

The enhanced ICIC can be considered as a good solution to solve the problem of ICI but it could result in unused subframes in some cases. So, in this paper we proposed an adaptive method that uses reduced power subframes which can change their power adaptively according to the user distance and consequently change the SINR. The edge users have the most benefit from this scheme as the scheduler focuses on those users. The results show that the average throughput of the users and edge users for both macro cells and pico cells have improved and the throughput for the whole network as well. There are many suggested further development for the proposed work, like adding the artificial intelligence to the system to decide which of the users should get the higher priority and not just relying on the SINR.

\section{REFERENCES}

[1] J. Wannstrom and K. Mallinson, "Heterogeneous networks in LTE-3GPP," 2014, [Online]. Available: https://www.3gpp.org/technologies/keywords-acronyms/1576-hetnet.

[2] Y.-C. Wang and S.-T. Chen, "Adaptive configuration of time-domain eICIC to support multimedia communications in LTE-A heterogeneous networks," 2017 IEEE 18th International Symposium on A World of Wireless, Mobile and Multimedia Networks (WoWMoM), Macau, 2017, pp. 1-6.

[3] 3GPP-TS22.220, "Service requirements for Home Node B and Home eNode B (Release 14)," 3rd Generation Partnership Project-TSG Services and System Aspects," 2017.

[4] L. Lindbom, R. Love, S. Krishnamurthy, C. Yao, N. Miki, and V. Chandrasekhar, "Enhanced inter-cell interference coordination for heterogeneous networks in lte-advanced: A survey," arXiv preprint arXiv:1112.1344, 2011.

[5] Q. Ye, B. Rong, Y. Chen, C. Caramanis, and J. G. Andrews, "Towards an optimal user association in heterogeneous cellular networks," 2012 IEEE Global Communications Conference (GLOBECOM), Anaheim, CA, USA, 2012, pp. 4143-4147.

[6] I. Guvenc, "Capacity and fairness analysis of heterogeneous networks with range expansion and interference coordination," IEEE Communications Letters, vol. 15, no. 10, pp. 1084-1087, 2011.

[7] A. B. A. Al-Aaloosi, "Inter-cell interference mitigation in LTE-advanced heterogeneous mobile networks," University of Salford, 2017.

[8] Samsung R1-111466, "Full Buffer Evaluation Results for CoMP Scenario 3 and 4," [Online]. Available: http://www.3gpp.org/ftp/tsg_ran/WG1_RL1/TSGR1_65/Docs/R1-111466.zip>

[9] J. Oh and Y. Han, "Cell selection for range expansion with almost blank subframe in heterogeneous networks," 2012 IEEE 23rd International Symposium on Personal, Indoor and Mobile Radio Communications - (PIMRC), Sydney, NSW, Australia, 2012, pp. 653-657.

[10] I. F. Akyildiz, D. M. Gutierrez-Estevez, R. Balakrishnan, and E. Chavarria-Reyes, "LTE-Advanced and the evolution to Beyond 4G (B4G) systems," Physical Communication, vol. 10, pp. 31-60, 2014.

[11] A. Daeinabi, K. Sandrasegaran, and X. Zhu, "Performance evaluation of cell selection techniques for picocells in LTE-advanced networks," 2013 10th International Conference on Electrical Engineering/Electronics, Computer, Telecommunications and Information Technology, Krabi, Thailand, 2013, pp. 1-6. 
[12] N. Naganuma, S. Nakazawa, S. Suyama, Y. Okumura, and H. Otsuka, "Adaptive control CRE technique for eICIC in HetNet," 2016 Eighth International Conference on Ubiquitous and Future Networks (ICUFN), Vienna, Austria, 2016, pp. 4-6.

[13] X. Gu, X. Deng, Q. Li, L. Zhang, and W. Li, "Capacity Analysis and Optimization in Heterogeneous Network with Adaptive Cell Range Control," International Journal of Antennas and Propagation, vol. 2014, 2014, Art. no. 215803, doi: 10.1155/2014/215803.

[14] "LTE; Evolved Universal Terrestrial Radio Access (E-UTRA) and Evolved Universal Terrestrial Radio Access Network (E-UTRAN); Overall description; Stage 2 (3GPP TS 36.300 version 14.2.0 Release 14)," 2017. [Online], Available: https://www.etsi.org/deliver/etsi_ts/136300_136399/136300/14.02.00_60/ts_136300v140200p.pdf

[15] Sabella, D., Rapone, D., Fodrini, M., Cavdar, C., Olsson, M., Frenger, P., and Tombaz, S., "Energy management in mobile networks towards 5G," Energy Management in Wireless Cellular and Ad-hoc Networks, vol. 50, pp. 397-427, 2016. doi: 10.1007/978-3-319-27568-0_17.

[16] K. I. Pedersen, Y. Wang, S. Strzyz, and F. Frederiksen, "Enhanced inter-cell interference coordination in cochannel multi-layer LTE-advanced networks," IEEE Wireless Communications, vol. 20, no. 3, pp. 120-127, 2013, doi: 10.1109/MWC.2013.6549291.

[17] R. Mendrzik, R. A. J. Castillo, G. Bauch, and E. Seidel, "Interference coordination-based downlink scheduling for heterogeneous LTE-A networks," 2016 IEEE Wireless Communications and Networking Conference, Doha, Qatar, 2016, pp. 1-6.

[18] B. Soret, H. Wang, K. I. Pedersen, and C. Rosa, "Multicell cooperation for LTE-advanced heterogeneous network scenarios," IEEE Wireless Communications, vol. 20, no. 1, pp. 27-34, 2013.

[19] A. Merwaday, S. Mukherjee, and I. Güvenç, "HetNet capacity with reduced power subframes," 2014 IEEE Wireless Communications and Networking Conference (WCNC), Istanbul, Turkey, 2014, pp. 1380-1385.

[20] G. R1-120023, "TSG-RAN WG1, Analysis of feasibility and standard impact of reduced power ABS," 2012, [Online], Available: https://www.3gpp.org/DynaReport/TDocExMtg--R1-68--29000.htm

[21] 3GPP R1-113635, "TSG-RAN WG1 "Performance evaluation of FeICIC with zero and reduced power ABS," 2011, [Online]. Available: https://www.3gpp.org/DynaReport/TDocExMtg--R1-67--28507.htm

[22] G. R1-121489, "Discussion on the features and signalling support for non-zero transmit power ABS," 3rd Genereation Partnership Project-TSG-RAN1, 2012.

[23] H. Hu, J. Weng, and J. Zhang, "Coverage performance analysis of FeICIC low-power subframes," IEEE Transactions on Wireless Communications, vol. 15, no. 8, pp. 5603-5614, 2016.

[24] H. Hu, J. Weng, J. Zhang, J. Zhang, and Y. Wang, "Modelling and analysis of reduced power subframes in two-tier femto HetNets," 2016 IEEE 83rd Vehicular Technology Conference (VTC Spring), Nanjing, China, 2016, pp. 1-5.

[25] T. Saito and F. Adachi, "De-centralized adaptive 2-step inter-cell interference coordination in distributed MIMO," 2018 24th Asia-Pacific Conference on Communications (APCC), Ningbo, China, 2018, pp. 224-228, doi: 10.1109/APCC.2018.8633471.

[26] R. A. Hassan, A. Idris, H. Adto, M. Ramadhan, and M. Kassim, "Reduction of Inter-Cell interference in close proximity cell using dynamic fractional frequency reuse method," 2017 IEEE Conference on Systems, Process and Control (ICSPC), Meleka, Malaysia, 2017, pp. 157-161.

[27] K. Fujisawa, F. Kemmochi, and H. Otsuka, "Personal-Cell Scheme Using Adaptive Control CRE for Multicarrier HetNets," 2019 IEEE 90th Vehicular Technology Conference (VTC2019-Fall), Honolulu, HI, USA, 2019, pp. 1-5, doi: 10.1109/VTCFall.2019.8891193.

[28] C.-N. Lee, J.-H. Lin, C.-F. Wu, M.-F. Lee, and F.-M. Yeh, "A dynamic CRE and ABS scheme for enhancing network capacity in LTE-advanced heterogeneous networks," Wireless Networks, vol. 25, pp. 3307-3322, 2019, doi: 10.1007/s11276-018-1723-2.

[29] A. Grassi, G. Piro, G. Boggia, M. Kurras, W. Zirwas, R. SivaSiva Ganesan, et al., "Massive MIMO interference coordination for 5G broadband access: Integration and system level study," Computer Networks, vol. 147, pp. 191-203, 2018.

[30] B. Soret, A. D. Domenico, S. Bazzi, N. H. Mahmood, and K. I. Pedersen, "Interference Coordination for 5G New Radio," IEEE Wireless Communications, vol. 25, no. 3, pp. 131-137, 2018, doi: 10.1109/MWC.2017.1600441.

[31] B. Furth and S. A. Ahson, "Long Term Evolution: 3GPP LTE Radio and Cellular Technology," Auerbach Publications, 2019.

[32] Y. G. El-Jaafreh, "Co-channel and Adjacent Channel Interference Calculations in Cellular Communications Systems," Journal of King Saud University - Engineering Sciences, vol. 12, no. 1, pp. 153-167, 2000, doi: 10.1016/S1018-3639(18)30711-6.

[33] X. Chu, D. Lopez-Perez, Y. Yang, and F. Gunnarsson, "Heterogeneous cellular networks: theory, simulation and deployment," Cambridge University Press, 2013.

[34] M. H. A. Khan, J.-G. Chung, and M. H. Lee, "Downlink performance of cell edge using cooperative BS for multicell cellular network," EURASIP Journal on Wireless Communications and Networking, vol. 56, 2016.

[35] E. Turgut and M. C. Gursoy, "Coverage in heterogeneous downlink millimeter wave cellular networks," IEEE Transactions on Communications, vol. 65, no. 10, pp. 4463-4477, 2017.

[36] G. Cong, "Pico Cell Densification Study in LTE Heterogeneous Networks," KTH:s Publikations database DiVa, 2012.

[37] 3GPP, "Evolved Universal Terrestrial Radio Access (E-UTRA); Radio Frequency (RF) system scenarios" 3GPP TR 36.942 v16.0.0 Release 16, 2020. 


\section{BIOGRAPHIES OF AUTHORS}
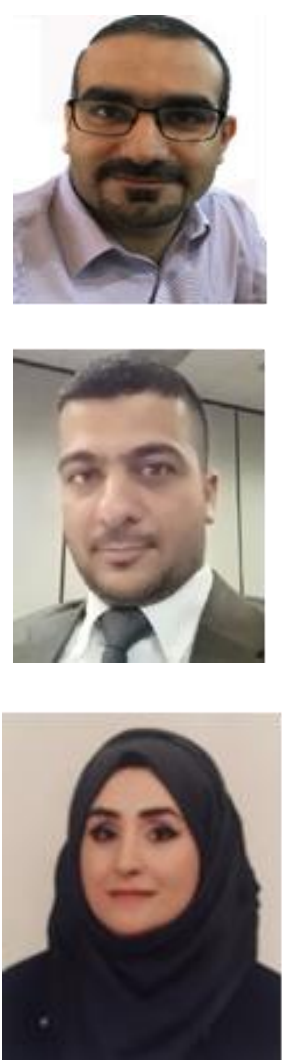

Mohammed Aal-Nouman, got his $\mathrm{PhD}$ degree in mobile communications from University of Salford- Manchester, UK in 2017. Since 2008 he is working in Al-Nahrain University, college of information engineering as a lecturer; teaching different subjects like mobile networks, wireless network, microprocessors, computer architecture and others. His research interest includes but not limited to Mobile and wireless communications, eHealth systems, Location-based services. Now he is affiliated in Department of Information and Communication Eng., College of Information Engineering. Al-Nahrain University, Baghdad, Iraq.

Osamah Abdullah has completed his $\mathrm{PhD}$ in electrical and computer engineering at Western Michigan University in December 2016. His teaching experience and practical skills are in communication systems and signal processing, alongside with his research background, his current research focuses on machine learning algorithms, UAV channel modeling, Brainwave signals detections, mobile communication, signal processing, and peer-to-peer/ad-hoc networking, especially with applications to group localization, tracking, and monitoring. Now he is affiliated in Department of Electrical Power Engineering Techniques. Al-Ma'moon University College, Baghdad, Iraq.

Email: osamah.abdullah@wmich.edu

Noor Qusay A. Al-Shaikhli has an MSc degree in Information \& Communication Engineering form Al-Nahrain University, Iraq in 2019, she got her B.Sc. degree from Greenwich University, UK \& MSA University, Egypt in Electronics \& Communication Engineering in 2011. Since 2013 working at Al-Nahrain University, College of Information Engineering and currently as an Assist. Lecturer. Research interest includes (but is not limited to) Antenna [Design, Simulation \& Fabrication], Wireless Communication \& FPGA, Mobile Communications \& 5G Communication. Now he is affiliated in Department of Information and Communication Engineering.College of Information Engineering. Al-Nahrain University. Baghdad, Iraq.

Email:noor.qusay@coie-nahrain.edu.iq 